\title{
SCATTERING THEORY AND ADIABATIC DECOMPOSITION OF THE $\zeta$-DETERMINANT OF THE DIRAC LAPLACIAN
}

\author{
Jinsung PARK* AND KRzysztof P. Wojciechowski
}

\begin{abstract}
In this note we announce the adiabatic decomposition formula for the $\zeta$-determinant of the Dirac Laplacian. Theorem 1.1 of this paper extends the result of our earlier work (see [8] and [9]), which covered the case of the invertible tangential operator. The presence of the non-trivial kernel of the tangential operator requires careful analysis of the small eigenvalues of the Dirac Laplacian, which employs elements of scattering theory.
\end{abstract}

\section{Statement of the Result}

Let $\mathcal{D}: C^{\infty}(M ; S) \rightarrow C^{\infty}(M ; S)$ denote a compatible Dirac operator acting on sections of a bundle of Clifford modules $S$ over a closed manifold $M$ of dimension $2 k+1$. Assume that we have a decomposition of $M$ as $M_{1} \cup M_{2}$, where $M_{1}$ and $M_{2}$ are compact manifolds with boundary so that

$$
M=M_{1} \cup M_{2} \quad, \quad M_{1} \cap M_{2}=Y=\partial M_{1}=\partial M_{2} .
$$

We assume that $M$ and the operator $\mathcal{D}$ have product structures in a neighborhood of the boundary $Y$. More precisely, we assume that there is a bicollar neighborhood $N=[-1,1] \times Y$ of $Y$ in $M$ such that both the Riemannian structure on $M$ and the Hermitian structure on $S$ are products when restricted to $N$. This implies that $\mathcal{D}$ has the following form when restricted to the submanifold $N$

$$
\mathcal{D}=G\left(\partial_{u}+B\right)
$$

Here $u$ denotes a normal variable, $G: S|Y \rightarrow S| Y$ is a bundle automorphism and $B$ is the corresponding Dirac operator on $Y$. Moreover, $G$ and $B$ do not depend on $u$ and they satisfy

$$
G^{*}=-G, G^{2}=-I d, B=B^{*} \text { and } G B=-B G .
$$

Received May 22, 2001.

*Partially supported by Korea Science and Engineering Foundation. 
The operator $B$ has a discrete spectrum with infinitely many positive and infinitely many negative eigenvalues. Let $\Pi_{>}\left(\right.$resp. $\left.\Pi_{<}\right)$denote the spectral projections onto the subspaces spanned by the eigensections of $B$ corresponding to the positive (resp. negative) eigenvalues and $\sigma_{1}, \sigma_{2}: \operatorname{ker} B \rightarrow \operatorname{ker} B$ denote the involutions of kernel of $B$ such that

$$
G \sigma_{i}=-\sigma_{i} G
$$

Let $\pi_{i}=\frac{I d-\sigma_{i}}{2}$ denote the orthogonal projections of the kernel of $B$ onto -1 eigenspace of $\sigma_{i}$. The orthogonal projections $P_{1}=\Pi_{<}+\pi_{1}$ and $P_{2}=\Pi_{>}+\pi_{2}$ provide elliptic self-adjoint boundary conditions for the operators $\mathcal{D}_{1}=\mathcal{D} \mid M_{1}$ and $\mathcal{D}_{2}=\mathcal{D} \mid M_{2}$ respectively. This means that the associated operators

$$
\left(\mathcal{D}_{i}\right)_{P_{i}}: \operatorname{dom}\left(\mathcal{D}_{i}\right)_{P_{i}} \rightarrow L^{2}\left(M_{i} ; S \mid M_{i}\right)
$$

with domains $\operatorname{dom}\left(\mathcal{D}_{i}\right)_{P_{i}}=\left\{s \in H^{1}\left(M_{i} ; S \mid M_{i}\right) ; P_{i}(s \mid Y)=0\right\}$ are self-adjoint Fredholm operators with $\operatorname{ker}\left(\left(\mathcal{D}_{i}\right)_{P_{i}}\right) \subset C^{\infty}\left(M_{i} ; S \mid M_{i}\right)$ and they both have discrete spectrum (see [1], [11]).

We now introduce the manifold $M_{R}$ equal to the manifold $M$ with $N$ replaced by $N_{R}=[-R, R] \times Y$ and $M_{1, R}, M_{1, \infty}, M_{2, R}, M_{2, \infty}$ which are manifolds $M_{1}$ or $M_{2}$ with the semicylinder $[0, R] \times Y,[0, \infty) \times Y$ or $[-R, 0] \times Y,(-\infty, 0] \times Y$ attached to them. Let $\mathcal{D}_{R}, \mathcal{D}_{i, R}, \mathcal{D}_{i, \infty}$ denote the natural extension of $\mathcal{D}$ to $M_{R}, M_{i, R}, M_{i, \infty}$ for $i=1,2$. We also use $\mathcal{D}_{1, \infty}, \mathcal{D}_{2, \infty}$ to denote the unique closed self-adjoint extension of those operators in the spaces $L^{2}\left(M_{1, \infty} ; S\right)$ and $L^{2}\left(M_{2, \infty} ; S\right)$. The operator $\mathcal{D}_{R}$ is a self-adjoint operator on $L^{2}\left(M_{R} ; S\right)$ and as such it has a discrete spectrum only. Analysis of the eigenvalues shows that they fall into three different categories. We have large eigenvalues $(l-v a l u e s)$ bounded away from 0 . Then there is infinitely many small eigenvalues $(s$-values $)$, which are of the size $O\left(\frac{1}{R}\right)$. Last, we have a finite amount of eigenvalues, which decay exponentially with $R$ (e-values). There exists $R_{0}$, such that for any $R>R_{0}$ number $h_{M}$ of $e$-values does not depend on $R$ and we have the formula

$$
h_{M}=\operatorname{dim}\left(\operatorname{ker}_{L^{2}}\left(\mathcal{D}_{1, \infty}\right)\right)+\operatorname{dim}\left(\operatorname{ker}_{L^{2}}\left(\mathcal{D}_{2, \infty}\right)\right)+\operatorname{dim}\left(L_{1} \cap L_{2}\right)
$$

(see [3], see also [6] and [12] for additional discussion). Here, $L_{i} \subset \operatorname{ker}(B)$ denotes the space of the extended $L^{2}$-solutions of $\mathcal{D}_{i, \infty}$.

We define a modified zeta function of $\mathcal{D}_{R}^{2}$ by the formula

$$
\zeta_{\mathcal{D}_{R}^{2}}(s)=\frac{1}{\Gamma(s)} \int_{0}^{\infty} t^{s-1} \operatorname{Tr}^{\prime}\left(e^{-t \mathcal{D}_{R}^{2}}\right) d t
$$

where $\operatorname{Tr}^{\prime}(\cdot)$ is taken over all the eigenvalues with the exception 
of $e$-values. The operators $\left(\mathcal{D}_{i, R}\right)_{P_{i}}$ do not have $e$-values (see [6]) and $h_{i}=\operatorname{dim}\left(\operatorname{ker}\left(\mathcal{D}_{i, R}\right)_{P_{i}}\right)$ is equal to

$$
\operatorname{dim}\left(\operatorname{ker}_{L^{2}}\left(\mathcal{D}_{i, \infty}\right)\right)+\operatorname{dim}\left(L_{i} \cap \operatorname{ker}\left(\sigma_{i}-1\right)\right)
$$

We define the zeta functions of $\left(\mathcal{D}_{i, R}\right)_{P_{i}}^{2}$ by

$$
\zeta_{\left(\mathcal{D}_{i, R}\right)_{P_{i}}^{2}}(s)=\frac{1}{\Gamma(s)} \int_{0}^{\infty} t^{s-1} \operatorname{Tr}^{\prime}\left(e^{-t\left(\mathcal{D}_{i, R}\right)_{P_{i}}^{2}}\right) d t
$$

where $\operatorname{Tr}^{\prime}(\cdot)$ is taken over the non-zero eigenvalues for $i=1,2$. The zeta functions $\zeta_{\mathcal{D}_{R}^{2}}(s), \zeta_{\left(\mathcal{D}_{i, R}\right)_{P_{i}}^{2}}(s)$ are regular at $s=0$ and we can define the $\zeta$-regularized determinants for these operators using the standard formula

$$
\ln \operatorname{det}_{\zeta} \mathfrak{D}_{R}^{2}=-\left.\frac{d}{d s}\left\{\zeta_{\mathfrak{D}_{R}^{2}}(s)\right\}\right|_{s=0}
$$

where $\mathfrak{D}_{R}^{2}$ denotes one of the aforementioned operators. In this announcement we study the following adiabatic limit

$$
\lim _{R \rightarrow 0} \frac{\operatorname{det}_{\zeta} \mathcal{D}_{R}^{2}}{\operatorname{det}_{\zeta}\left(\mathcal{D}_{1, R}\right)_{P_{1}}^{2} \cdot \operatorname{det}_{\zeta}\left(\mathcal{D}_{2, R}\right)_{P_{2}}^{2}}
$$

We have to introduce elements of Scattering Theory in order to present the formula for the limit (1.6). The operators $\mathcal{D}_{i, \infty}^{2}$ over $M_{i, \infty}$ have continuous spectrum equal to $[0, \infty)$. The number $\lambda \in[0, \infty)$ and $\phi \in \operatorname{ker}(B)$ determine a generalized eigensection of $\mathcal{D}_{1, \infty}$, which has the following form on $[0, \infty) \times Y \subset$ $M_{1, \infty}($ see $(4.24)$ in $[6])$

$$
E(\phi, \lambda)=e^{-i \lambda u}(\phi-i G \phi)+e^{i \lambda u} C_{1}(\lambda)(\phi-i G \phi)+\theta(\phi, \lambda)
$$

where $\theta(\phi, \lambda)$ is a square integrable section of $S$ on $M_{1, \infty}$ which is orthogonal to $\operatorname{ker}(B)$, when restricted to $\{u\} \times Y$, and $C_{1}(\lambda)$ is the scattering matrix. There is also corresponding scattering matrix $C_{2}(\lambda)$ determined by the operator $\mathcal{D}_{2, \infty}$ over $M_{2, \infty}$. We refer to [6] and [7] (see also [5]) for the presentation of the necessary material from Scattering Theory.

Let $C: W \rightarrow W$ denote a unitary operator acting on the finite dimensional vector space $W$. We introduce the operator $D(C)$ equal to the differential operator $-i \frac{1}{2} \frac{d}{d u}$ acting on $L^{2}\left(S^{1}, E_{C}\right)$ where $E_{C}$ is the flat vector bundle over $S^{1}=\mathbb{R} / \mathbb{Z}$ with the complex conjugate of $C$ as the holonomy group. We define operators

$$
I_{1}=(G+i): \operatorname{ker}(B) \rightarrow \operatorname{ker}(G-i)
$$




$$
\begin{gathered}
I_{2}=(G-i): \operatorname{ker}(B) \rightarrow \operatorname{ker}(G+i) \\
P_{\sigma_{i}}=\frac{1}{2}\left(\sigma_{i}-1\right): \operatorname{ker}(B) \rightarrow \operatorname{ker}\left(\sigma_{i}+1\right)
\end{gathered}
$$

and

$$
S_{\sigma_{i}}(\lambda)=-\left.P_{\sigma_{i}} \circ C_{i}(\lambda) \circ I_{i}\right|_{\operatorname{ker}\left(\sigma_{i}+1\right)}: \operatorname{ker}\left(\sigma_{i}+1\right) \rightarrow \operatorname{ker}\left(\sigma_{i}+1\right)
$$

Then $C_{12}:=\left.C_{1}(0) \circ C_{2}(0)\right|_{\operatorname{ker}(G+i)}, S_{\sigma_{1}}:=S_{\sigma_{1}}(0)$ and $S_{\sigma_{2}}:=S_{\sigma_{2}}(0)$ are the unitary operators acting on the finite dimensional vector spaces and we have well-defined self-adjoint, elliptic operators $D\left(C_{12}\right), D\left(S_{\sigma_{1}}\right), D\left(S_{\sigma_{2}}\right)$. Now, we are ready to state the main result

Theorem 1.1. The following formula holds:

$$
\lim _{R \rightarrow \infty} \frac{R^{2 h} \cdot \operatorname{det}_{\zeta} \mathcal{D}_{R}^{2}}{\operatorname{det}_{\zeta}\left(\mathcal{D}_{1, R}\right)_{P_{1}}^{2} \cdot \operatorname{det}_{\zeta}\left(\mathcal{D}_{2, R}\right)_{P_{2}}^{2}}=\frac{2^{-\zeta_{B^{2}}(0)} \cdot \operatorname{det}_{\zeta} \frac{1}{4} D\left(C_{12}\right)^{2}}{\operatorname{det}_{\zeta} D\left(S_{\sigma_{1}}\right)^{2} \cdot \operatorname{det}_{\zeta} D\left(S_{\sigma_{2}}\right)^{2}}
$$

where $h=h_{M}-h_{1}-h_{2}=\operatorname{dim}\left(L_{1} \cap L_{2}\right)-\operatorname{dim}\left(L_{1} \cap \operatorname{ker}\left(\sigma_{1}-1\right)\right)-\operatorname{dim}\left(L_{2} \cap\right.$ $\left.\operatorname{ker}\left(\sigma_{2}-1\right)\right)$ and $P_{1}=\Pi_{<}+\pi_{\sigma_{1}}, P_{2}=\Pi_{>}+\pi_{\sigma_{2}}$.

Remark 1.2. (1) A special case of the Theorem 1.1 was proved in [8], [9], where it was assumed that

$$
\operatorname{ker}_{L^{2}} \mathcal{D}_{1, \infty}=\operatorname{ker}_{L^{2}} \mathcal{D}_{2, \infty}=\operatorname{ker} B=\{0\}
$$

Assumption (1.8) implies the vanishing of all $s$-values and $e$-values, in other words, all eigenvalues of all operators involved are bounded away from 0 . This reduces formula (1.7) to the equality

$$
\lim _{R \rightarrow \infty} \frac{\operatorname{det}_{\zeta} \mathcal{D}_{R}^{2}}{\operatorname{det}_{\zeta} \mathcal{D}_{1, R, \Pi_{<}}^{2} \cdot \operatorname{det}_{\zeta} \mathcal{D}_{2, R, \Pi_{>}}^{2}}=2^{-\zeta_{B^{2}}(0)} .
$$

(2) The main issue in the present work is the analysis of $s$-values. The proof of Theorem 1.1 uses some ideas of the work in W. Müller (see [6]). 


\section{Scattering Matrix and Slowly Decaying Eigenvalues}

In this section we investigate the relation between the scattering matrices $C_{1}(\lambda), C_{2}(\lambda)$ and the $s$-values of the operators $\mathcal{D}_{R},\left(\mathcal{D}_{1, R}\right)_{P_{1}}$ and $\left(\mathcal{D}_{2, R}\right)_{P_{2}}$.

Let $\varphi$ be a eigensection of $\mathcal{D}_{R}$, which corresponds to $s$-values $\lambda=\lambda(R)$, that is

$$
\mathcal{D}_{R} \varphi=\lambda \varphi \quad \text { with } \quad|\lambda|<R^{-\kappa}
$$

for some fixed $\kappa$ with $0<\kappa<1$. Now we study the $s-$ values of the operator $\mathcal{D}_{R}$ over $M_{R}$. We introduce the manifold

$$
\bar{M}_{R}=M_{1, R} \sqcup M_{2, R}
$$

The boundary of $\bar{M}_{R}$ is equal to the sum of two copies of $Y$. We consider $\mathcal{D}_{R}$, the operator on a closed manifold $M_{R}$, as the Dirac operator on $\bar{M}_{R}$, which satisfies the transmission boundary condition. In particular, the corresponding eigensection $\varphi$ to $s$-values $\lambda=\lambda(R)$ satisfies the "transmission boundary condition"

$$
\left.\varphi\right|_{\partial M_{1, R}}=\left.\varphi\right|_{\partial M_{2, R}}
$$

We refer to [2] for more detailed discussion of the transmission problem (see also $[8]$ ). We want to warn the reader that, when we discuss transmission boundary condition, it would be natural to consider $M_{1, R}$ as $M_{1, R}=M_{1} \cup[-R, 0] \times Y$, but in the following we parametrize cylindrical parts as

$$
M_{1, R}=M_{1} \cup[0, R] \times Y \quad, \quad M_{2, R}=M_{2} \cup[-R, 0] \times Y .
$$

The section $\varphi$ can be represented in the following way on $[0, R] \times Y \subset M_{1, R}$

$$
\varphi=e^{-i \lambda u} \psi_{1}+e^{i \lambda u} \psi_{2}+\varphi_{1}
$$

where $\psi_{1} \in \operatorname{ker}(G-i), \psi_{2} \in \operatorname{ker}(G+i)$ and $\varphi_{1}$ is orthogonal to $\operatorname{ker} B$ when restricted to $\{u\} \times Y$. The eigenvalue $\lambda(R)$ is not bounded away from 0 , hence $\psi_{1}, \psi_{2}$ are non-trivial sections of $S \mid Y$ (see Theorem 2.2 in [12]). Choose $\phi \in L_{1}$ such that $\psi_{1}=\phi-i G \phi$. Then the generalized eigensection $E(\phi, \lambda)$ associated to $\phi$ is given by

$$
E(\phi, \lambda)=e^{-i \lambda u}(\phi-i G \phi)+e^{i \lambda u} C_{1}(\lambda)(\phi-i G \phi)+\theta(\phi, \lambda) .
$$

Following [6], we introduce $F=\left.\varphi\right|_{M_{1, R}}-\left.E(\phi, \lambda)\right|_{M_{1, R}}$. We know that there exist positive constants $c_{1}, c_{2}$, such that

$$
\left.|| \varphi_{1}\right|_{\{R\} \times Y}||<c_{1} e^{-c_{2} R}
$$


(see for instance Lemma 2.1 in [12]). Green's Theorem gives us

$$
0=\langle D F, F\rangle_{M_{1, R}}-\langle F, D F\rangle_{M_{1, R}}=\int_{Y}\langle G F, F\rangle d y
$$

This leads to

$$
\int_{Y}\langle G F, F\rangle d y=-i\left\|C_{1}(\lambda) \psi_{1}-\psi_{2}\right\|^{2}+O\left(e^{-c R}\right)
$$

for some positive constant $c$, and we get the following inequality

$$
\left\|C_{1}(\lambda) \psi_{1}-\psi_{2}\right\|^{2}<c_{1} e^{-c_{2} R}
$$

for some constants $c_{1}, c_{2}$ (compare [6]). Similarly, we have

$$
\varphi=e^{i \lambda u} \psi_{3}+e^{-i \lambda u} \psi_{4}+\varphi_{2}
$$

over $[-R, 0] \times Y \subset M_{2, R}$, where $\psi_{3} \in \operatorname{ker}(G+i), \psi_{4} \in \operatorname{ker}(G-i)$ and $\varphi_{2}$ is orthogonal to $\operatorname{ker}(B)$ when restricted to $\{u\} \times Y$. Again we have the expected estimate

$$
\left\|C_{2}(\lambda) \psi_{3}-\psi_{4}\right\|^{2}<c_{1} e^{-c_{2} R}
$$

The transmission boundary condition over $Y \sqcup Y=\partial M_{1, R} \sqcup \partial M_{2, R}$ implies the equalities

$$
e^{-i \lambda R} \psi_{1}+e^{i \lambda R} \psi_{2}=e^{-i \lambda R} \psi_{3}+e^{i \lambda R} \psi_{4}
$$

so that

$$
\psi_{1}=e^{2 i \lambda R} \psi_{4}, \quad \psi_{3}=e^{2 i \lambda R} \psi_{2}
$$

By (2.1), (2.2), (2.3), we have

$$
\begin{gathered}
\left\|e^{2 i \lambda R} C_{1}(\lambda) \psi_{4}-\psi_{2}\right\|^{2}<c_{1} e^{-c_{2} R} \\
\left\|e^{2 i \lambda R} C_{2}(\lambda) \psi_{2}-\psi_{4}\right\|^{2}<c_{1} e^{-c_{2} R} .
\end{gathered}
$$

We combine these inequalities and obtain

$$
\begin{gathered}
\left\|e^{4 i \lambda R} C_{1}(\lambda) \circ C_{2}(\lambda) \psi_{2}-\psi_{2}\right\|^{2}<c_{3} e^{-c_{4} R} \\
\left\|e^{4 i \lambda R} C_{2}(\lambda) \circ C_{1}(\lambda) \psi_{4}-\psi_{4}\right\|^{2}<c_{3} e^{-c_{4} R}
\end{gathered}
$$


for some positive constants $c_{3}, c_{4}$. The unitary operator $C_{1}(\lambda) \circ C_{2}(\lambda)$ is an analytic function of $\lambda$ for sufficiently small $\lambda$. This whole analysis follows the method presented in [6] and results in the following Proposition.

Proposition 2.1. There exists $R_{0}$ such that for $R>R_{0}$ the $s-$ value $\lambda(R)$ of $\mathcal{D}_{R}$ satisfies

$$
4 R \lambda(R)+\alpha_{j}(\lambda(R))=2 \pi k+O\left(e^{-c R}\right)
$$

for an integer $k$ with $|k|<R^{1-\kappa}$, where $\exp \left(i \alpha_{j}(\lambda(R))\right)$ is an eigenvalue of the restriction of the unitary operator $C_{1}(\lambda(R)) \circ C_{2}(\lambda(R))$ to $\operatorname{ker}(G+i) \subset \operatorname{ker}(B)$.

Remark 2.2. The map $C_{1}(0) \circ C_{2}(0)$ on $\operatorname{ker}(G+i)$ is not the identity map. However, it is equal to the identity, when restricted to the subspace $I_{2}\left(L_{1} \cap L_{2}\right)$, where

$$
I_{2}=(G-i): \operatorname{ker}(B) \rightarrow \operatorname{ker}(G+i)
$$

It follows that the number of $j$ 's such that $\alpha_{j}(0)=0$ is equal to $\operatorname{dim}\left(L_{1} \cap L_{2}\right)$. This is the dimension of the space of eigensections corresponding to $e$-values, which are not determined by $\operatorname{ker}_{L^{2}}\left(\mathcal{D}_{i, \infty}\right)$.

Similarly, we have the corresponding analysis for the slowly decaying eigenvalues of the operators $\left(\mathcal{D}_{i, R}\right)_{P_{i}}$ for $i=1,2$.

Proposition 2.3. There exists $R_{0}$ such that for $R>R_{0}$ the $s-$ value $\lambda=\lambda(R)$ of $\left(\mathcal{D}_{i, R}\right)_{P_{i}}$ satisfies

$$
2 R \lambda(R)+\beta_{j}(\lambda(R))=2 \pi k+O\left(e^{-c R}\right)
$$

for an integer $k$ with $|k|<R^{1-\kappa}$, and $\exp \left(i \beta_{j}(\lambda(R))\right)$ an eigenvalue of the unitary operator $S_{\sigma_{i}}(\lambda(R))=-P_{\sigma_{i}} \circ C_{i}(\lambda(R)) \circ I_{\sigma_{i}}: \operatorname{ker}\left(\sigma_{i}+1\right) \rightarrow \operatorname{ker}\left(\sigma_{i}+1\right)$ and $i=1,2$.

Remark 2.4. The map $S_{\sigma_{i}}(0)$ restricted to the subspace $\operatorname{ker}\left(\sigma_{i}+1\right) \cap$ $\operatorname{ker}\left(C_{i}(0)+1\right) \subset \operatorname{ker}\left(\sigma_{i}+1\right)$ is equal to $I$, and the number of $j$ 's such that $\beta_{j}(0)=0$ is equal to $\operatorname{dim}\left(\operatorname{ker}\left(\sigma_{\mathrm{i}}+1\right) \cap \operatorname{ker}\left(\mathrm{C}_{\mathrm{i}}(0)+1\right)\right)$. This is the number of zero eigenvalues of $\left(\mathcal{D}_{i, R}\right)_{P_{i}}$ which are not in $\operatorname{ker}_{L^{2}}\left(\mathcal{D}_{i, \infty}\right)$ for $i=1,2$. 


\section{Sketch of the Proof of Theorem 1.1}

In this section we briefly sketch the proof of Theorem 1.1. We refer to [10] for the detailed exposition.

We define

$$
\zeta_{r e l, R}(s):=\frac{1}{\Gamma(s)} \int_{0}^{\infty} t^{s-1}\left[\operatorname{Tr}\left(e^{-t \mathcal{D}_{R}^{2}}-e^{-t\left(\mathcal{D}_{1, R}\right)_{P_{1}}^{2}}-e^{-t\left(\mathcal{D}_{2, R}\right)_{P_{2}}^{2}}\right)-h\right] d t
$$

where $h=\operatorname{dim}\left(L_{1} \cap L_{2}\right)-\operatorname{dim}\left(L_{1} \cap \operatorname{ker}\left(\sigma_{1}-1\right)\right)-\operatorname{dim}\left(L_{2} \cap \operatorname{ker}\left(\sigma_{2}-1\right)\right)$. We decompose $\zeta_{r e l, R}(s)$ into two parts

$$
\zeta_{s}^{R}(s)=\frac{1}{\Gamma(s)} \int_{0}^{R^{2-\varepsilon}}(\cdot) d t \quad, \quad \zeta_{l}^{R}(s)=\frac{1}{\Gamma(s)} \int_{R^{2-\varepsilon}}^{\infty}(\cdot) d t
$$

where $0<\epsilon<1$. The derivatives of $\zeta_{s}^{R}(s), \zeta_{l}^{R}(s)$ at $s=0$ give the small and large time contribution. The standard computation shows that

$\left(\zeta_{s}^{R}\right)^{\prime}(0)=\int_{0}^{R^{2-\epsilon}} t^{-1} \operatorname{Tr}\left(e^{-t \mathcal{D}_{R}^{2}}-e^{-t\left(\mathcal{D}_{1, R}\right)_{P_{1}}^{2}}-e^{-t\left(\mathcal{D}_{2, R}\right)_{P_{2}}^{2}}\right) d t+h \gamma-h(2-\epsilon) \log R$,

where $\gamma$ denotes the Euler constant. We analyze the "small time" contribution

$$
\int_{0}^{R^{2-\epsilon}} t^{-1}\left[\operatorname{Tr}\left(e^{-t \mathcal{D}_{R}^{2}}-e^{-t\left(\mathcal{D}_{1, R}\right)_{P_{1}}^{2}}-e^{-t\left(\mathcal{D}_{2, R}\right)_{P_{2}}^{2}}\right)-h\right] d t
$$

using a method developed in [4] and explicit computations made in [10]. It follows that

$$
\lim _{R \rightarrow \infty}\left(\left.\frac{d}{d s} \zeta_{s}^{R}(s)\right|_{s=0}+h(2-\varepsilon) \cdot \ln R\right)=-\ln 2 \cdot \zeta_{B^{2}}(0)+h \gamma
$$

We have the equality

$$
\left(\zeta_{l}^{R}\right)^{\prime}(0)=\int_{R^{-\epsilon}}^{\infty} t^{-1}\left[\operatorname{Tr}\left(e^{-t R^{2} \mathcal{D}_{R}^{2}}-e^{-t R^{2}\left(\mathcal{D}_{1, R}\right)_{P_{1}}^{2}}-e^{-t R^{2}\left(\mathcal{D}_{2, R}\right)_{P_{2}}^{2}}\right)-h\right] d t
$$

and the analysis of $s$-values from Section 2 (see Proposition 2.1 and Proposition 2.3) provides the proof of the following result. The details will appear in [10].

\section{Theorem 3.1.}

$$
\begin{aligned}
& \lim _{R \rightarrow \infty} \int_{R^{-\epsilon}}^{\infty} t^{-1}\left[\operatorname{Tr}\left(e^{-t R^{2} \mathcal{D}_{R}^{2}}-e^{-t R^{2}\left(\mathcal{D}_{1, R}\right)_{P_{1}}^{2}}-e^{-t R^{2}\left(\mathcal{D}_{2, R}\right)_{P_{2}}^{2}}\right)-h\right] d t+h \gamma+h \epsilon \log R \\
& =\left.\frac{d}{d s}\right|_{s=0}\left\{\frac{1}{\Gamma(s)} \int_{0}^{\infty} t^{s-1}\left[\operatorname{Tr}\left(e^{-t \frac{1}{4} D\left(C_{12}\right)^{2}}-e^{-t D\left(S_{\sigma_{1}}\right)^{2}}-e^{-t D\left(S_{\sigma_{2}}\right)^{2}}\right)-h\right] d t\right\}
\end{aligned}
$$

This ends the proof of Theorem 1.1. 


\section{References}

[1] Booß-Bavnbek, B. and Wojciechowski, K.P., Elliptic Boundary Problems for Dirac Operators, Mathematics: Theory \& Applications. Birkhäuser Boston, Inc., Boston, MA, 1993.

[2] Brünning, J. and Lesch, M., On the $\eta$-invariant of certain nonlocal boundary value problems, Duke Math. J. 96 (1999) no. 2, 425-468.

[3] Cappell, S.E., Lee, R. and Miller, E.Y., Self-adjoint elliptic operators and manifold decompositions. I. Low eigenmodes and stretching, Comm. Pure Appl. Math. 49 (1996), no. 8, 825-866.

[4] Douglas, R.G. and Wojciechowski, K.P., Adiabatic limits of the $\eta$-invariants. The odddimensional Atiyah-Patodi-Singer problem, Comm. Math. Phys. 142 (1991), no. 1, 139168.

[5] Melrose, R.B., Geometric scattering theory. Stanford Lectures. Cambridge University Press, Cambridge, 1995.

[6] Müller, W., Eta invariants and manifolds with boundary, J. Differential Geom. 40 (1994), no. 2, 311-377.

[7] Müller, W., Relative zeta functions, relative determinants, and scattering theory, Comm. Math. Phys. 192 (1998), no. 2, 309-347.

[8] Park, J. and Wojciechowski, K. P., Relative $\zeta$-determinant and Adiabatic decomposition of the $\zeta$-determinat of the Dirac Laplacian, Lett. Math. Phys. 52 (2000), no. 4, 329-337.

[9] Park, J. and Wojciechowski, K. P., Adiabatic decomposition of the $\zeta$-determinant of the Dirac Laplacian I. The case of invertible tangential operator, to appear in Comm. Partial Differential Equations.

[10] Park, J. and Wojciechowski, K. P., Scattering Theory and the Adiabatic Decomposition of the $\zeta$-determinant of the Dirac Laplacian, preprint.

[11] Seeley, R. T., Topics in pseudodifferential operators, 1969 Pseudo-Diff. Operators (C.I.M.E., Stresa, 1968) 167-305 Edizioni Cremonese, Rome.

[12] Wojciechowski, K. P., The additivity of the $\eta$-invariant: the case of an invertible tangential operator, Houston J. Math. 20 (1994), no. 4, 603-621.

Department of Mathematics, IUPUi (Indiana/Purdue), Indianapolis, IN 462023216, U.S.A.

E-mail address: jinspark@indiana.edu

E-mail address: kwojciechowski@math.iupui.edu 